\title{
Hidden Magnetic Structures Revealed
}

\section{Researchers capture the previously hidden twists of spins in the domain wall of a "proper" antiferromagnetic material, chromium oxide.}

\section{By Katherine Wright}

6 "Interesting but useless," is how Louis Néel famously described antiferromagnets, materials for whose discovery he was awarded the 1970 Nobel Prize in physics. Jump forward 50 years and these materials are trending among condensed-matter physicists, who are exploring their use in next-generation information-processing and storage devices.

But to take the step from useless to useful, many unknowns still need to be uncovered. Now Martin Wörnle and his colleagues at the Swiss Federal Institute of Technology (ETH) in Zurich resolve one of those mysteries: how the spins in a "proper"

antiferromagnetic material-one where the spins can only point either up or down-twist between domains [1].

The team used a technique called nanoscale scanning diamond magnetometry, which can measure magnetic fields of just a few microtesla with a spatial resolution of less than $50 \mathrm{~nm}$, to map the stray magnetic field for different samples of chromium oxide. The stray magnetic field is the field that protrudes from a material, and it can be used to infer the orientation of spins

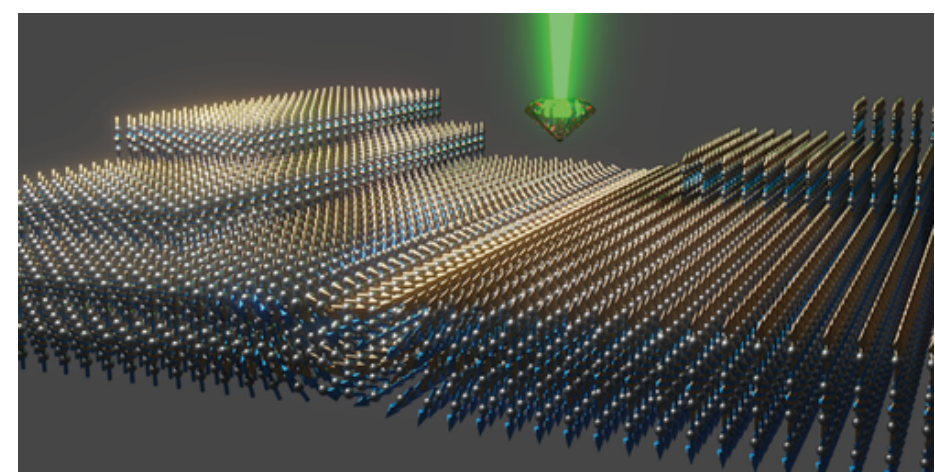

Credit: M. Wörnle/ETH Zurich inside the domain walls.

Wörnle and his colleagues observed two types of domain wall. In Bloch-type walls, the spin direction rotates smoothly in the plane of the domain wall, passing the midway point at the center of the wall. In Néel-type walls, the pointing direction is perpendicular to the wall, and the spins have a chiral twist. Most samples contained the energetically favored Bloch-type walls, which have a smaller stray magnetic field. Other researchers have captured similar images for antiferromagnets in which the spins can point in more than two directions, but the team notes that these are the first images for a bulk sample of a proper antiferromagnet.

Katherine Wright is the Deputy Editor for Physics.

\section{REFERENCES}

1. M. Wörnle et al., "Coexistence of Bloch and Néel walls in a collinear antiferromagnet," Phys. Rev. B 103, 094426 (2021). 